\title{
Endorectal power Doppler/grayscale ultrasound-guided biopsies vs. multiparametric MRI/ultrasound fusion-guided biopsies in males with high risk of prostate cancer: A prospective cohort study
}

\author{
ZHIZHONG HE ${ }^{1}$, ZHIFAN YUAN ${ }^{1}$, LIMEI LIANG ${ }^{1}$, XINX IN XIE $^{2}$, \\ JIANJUN YUAN ${ }^{1}$, WENXU HE ${ }^{1}$, JUNJUN CHEN ${ }^{1}$ and YONGPEI KUANG ${ }^{1}$ \\ ${ }^{1}$ Department of Ultrasound, Dongguan People's Hospital Affiliated to Southern Medical University, \\ Dongguan, Guangdong 523000; ${ }^{2}$ Department of Ultrasound, The First Affiliated Hospital of Anhui Medical University, \\ Hefei, Anhui 230022, P.R. China
}

Received February 19, 2019; Accepted August 30, 2019

DOI: $10.3892 / \mathrm{etm} .2019 .8151$

\begin{abstract}
Multiparametric MRI fusion with transrectal ultrasound (mpMRI/TRUS)-guided biopsy has the sensitivity of mpMRI with the practicality of TRUS, but males with no cancerous lesion(s) detected on mpMRI have a considerable remaining risk of cancer. Endorectal power Doppler ultrasound improves the sensitivity of grayscale ultrasound-guided biopsies. The objective of the present study was to evaluate the beneficial effect of endorectal power Doppler/grayscale ultrasound-guided biopsy over that of mpMRI/TRUS-guided biopsy for decision-making regarding prostatectomy in males with a high risk of prostate cancer. Data regarding endorectal power Doppler/grayscale ultrasound-guided biopsies and mpMRI/TRUS-guided biopsies of 1,094 males with elevated specific prostate antigen, were included. Radical prostatectomy was performed in males aged $<70$ years with Gleason scores $\geq 3+4$ in any one of the biopsy reports. The histopathological data of the surgical specimen of 776 males were included in the analysis. Compared to the histopathology of the surgical specimen, endorectal power Doppler/grayscale ultrasound-guided biopsies had a lower sensitivity (0.930 vs. 1.000; $\mathrm{P}<0.0001)$ but mpMRI/TRUS-guided biopsies had the same sensitivity ( 0.990 vs. $1.000 ; \mathrm{P}=0.02)$. The accuracy of mpMRI/TRUS-guided biopsies was higher than that of endorectal power Doppler/grayscale ultrasound-guided biopsies (0.944 vs. 0.783). On mpMRI, lesions of 105
\end{abstract}

Correspondence to: Dr Yongpei Kuang, Department of Ultrasound, Dongguan People's Hospital Affiliated to Southern Medical University, 3 Kita Suminoe New Valley Chung Wan Road South, Dongguan, Guangdong 523000, P.R. China

E-mail: yongpeikuang@hotmail.com

Key words: grayscale ultrasound, targeted biopsy, power Doppler, prostate cancer, prostatectomy subjects $(10 \%)$ with a Likert scale score of $<3$ were identified. Among them, 14 subjects $(2 \%)$ had Gleason scores of $\geq 3+4$ as determined by endorectal power Doppler/grayscale ultrasound-guided biopsies. In addition, 20 (2\%) false-positive lesions compared to the histopathological analysis of the surgical specimen were identified from mpMRI/TRUS-guided biopsies. In conclusion, mpMRI/TRUS-guided biopsy was indicated to have a moderate performance and endorectal power Doppler/grayscale ultrasound-guided biopsy had a scant performance for decision-making regarding prostatectomy.

\section{Introduction}

In Europe and in the US, prostate cancer is the most common cancer type in males (1). The occurrence of prostate cancer varies among different ethnicities (2). In Chinese males, prostate cancer has an incidence of 121 in 1,000,000 males (2) and it is the fifth leading cause of cancer-associated mortality in males (3). Mortality due to prostate cancer may be reduced by proper diagnosis (4). Radiological images have a crucial role in the diagnosis of prostate cancer (5). Additionally, serum prostate-specific antigen testing is frequently performed following biopsy due to the high frequency in the elevation of prostate-specific antigen expression in patients with prostate cancer (6).

Prostate-specific antigen is produced by the prostate but is not a prostate cancer biomarker. It may also be altered during inflammation or infection, as well as in benign prostatic hyperplasia (6). In Chinese males, it is also associated with obesity (7). Urologists in China have put rigorous effort in improving the quality of screening and treatment of cancer of the prostate gland with radiological methods, e.g. MRI, CT, transrectal ultrasound (TRUS), and positron emission tomography (8). Among the radiological methods, TRUS provides more appropriate details than MRI and CT (5). Multiparametric MRI (mpMRI) has value in the diagnosis of prostate cancer (8) but it is only able to diagnose localized prostate cancer with a volume of $\geq 0.2 \mathrm{ml}$ (9). A prostate biopsy 
is performed in order to discover a prostate cancer in the case of persistent altered prostate-specific antigen level and/or in the case of suspicion warranting digital rectal examination (10). mpMRI fused with TRUS (mpMRI/TRUS)-guided biopsy has the sensitivity of mpMRI (11) with the practicality of TRUS (12) and is a promising method (13) for prostate cancer diagnosis (14). However, to overcome inaccuracies associated with the biopsy technique, mpMRI/TRUS fusion biopsies require a high volume of samples (14). Subjects with undetected cancerous lesion(s) on mpMRI still have a certain probability of having prostate cancer (15) but mpMRI/TRUS is not performed for those.

The utility of power Doppler with grayscale ultrasound is used in the detection of prostate cancer (5). Endorectal power Doppler ultrasound may detect capsular extension, visualize tumor vascularity and improve the sensitivity of grayscale ultrasound-guided biopsies (16). All of these pre-operative data should be utilized by the surgeon to perform the best radical prostatectomy technique in order to obtain the best achievable functional result $(17,18)$.

The objective of the present prospective study was to evaluate the beneficial score of endorectal power Doppler combined with grayscale ultrasound-guided biopsy over that of mpMRI/TRUS-guided biopsy for decision making regarding prostatectomy in Chinese males with a high risk of prostate cancer.

\section{Materials and methods}

Materials. Levofloxacin (Loxof) was purchased from Ranbaxy Pharmaceuticals Pvt. Ltd (Sun Pharmaceutical Industries, Ltd.). Lidocaine jelly (Xylocaine gel) was purchased from AstraZeneca Pharmaceuticals Co. Ltd. Diclofenac (50 mg) with paracetamol $(500 \mathrm{mg})$ tablet (Lederflam Forte) was purchased from Wyeth, Inc. The loose enema was purchased from Torrent Pharmaceuticals, Ltd.

Inclusion criteria. Between January 2013 and February 2019, a total of 1,215 males aged $\geq 40$ years with complaints including weak flow during urination, difficulties to start urinating, weak flow of urine and a sensation of improper urinary bladder emptying were available at an outpatient setting of Dongguan People's Hospital Affiliated to Southern Medical University (Dongguan, China) and the First Affiliated Hospital of Anhui Medical University (Anhui, China) were included in the present study (19). Males with elevated prostate-specific antigen (normal range, $\leq 3.0 \mathrm{ng} / \mathrm{ml}$ for subjects $<50$ years, $\leq 3.5 \mathrm{ng} / \mathrm{ml}$ for $50-59$ years, $\leq 4.5 \mathrm{ng} / \mathrm{ml}$ for $60-69$ years and $\leq 5.5 \mathrm{ng} / \mathrm{ml}$ for $\geq 70$ years) (20) or abnormal structure of the prostate on palpitation on rectal examination (hard, lumpy or enlarged prostate) under digital rectal examination (19) were included in the study.

Exclusion criteria. Males with confirmed prostate cancer, negative biopsies for prostate cancer in the past 6 months, normal prostate-specific antigen levels, normal rectal examinations, men with normal prostate-specific antigen levels and abnormal prostate palpitations (considered inflammation of the prostate and not prostate cancer), a Gleason score of $3+3$ and age of $<40$ years were excluded from the study. Although patients with age $>70$ years were not recommended for prostatectomy, they were also subjected to biopsies.

mpMRI. Males were examined with an MRI 3.0 Tesla (AIRIS Vento O5 0.3T; Hitachi Aloka, Medical, Ltd.) using phased-array torso coils (16 elements, Hitachi Aloka Medical, Ltd.) and with an endorectal coil. Dynamic contrast-enhanced imaging, diffusion-weighted imaging with b-values of $>1,500$, apparent diffusion coefficient value imaging and multiplanar T2-weighted imaging (T2WI) scans were performed for all patients (21). MR images were analyzed with the Prostate Imaging Reporting and Data System version 1 (22). Based on the overall impression of the prostate in MR images, each lesion was assigned a Likert scale score (five-point scale method: Unlikely benign, 1; most probably benign, 2; equivocal prostate cancer, 3 ; probably malignant, 4 ; and highly suspicious of malignancy, 5 (23). Males with lesions with a Likert scale score of $\geq 3$ were subjected to mpMRI/TRUS-guided biopsies (according to the institutional guidelines for prostate cancer examinations) (23). Five radiologists (with a minimum of 3 years of experience) of the institute(s) performed mpMRI and assigned the Likert scale score to each of the lesions. All enrolled patients were subjected to both types of biopsies, which were performed sequentially.

Endorectal power Doppler/grayscale ultrasound-guided biopsy. The patients were instructed to lie down in the left lateral decubitus position with flexed knees and hips. Rectal lidocaine jelly was applied to the rectal probe and the latex cuff. The prostate gland was examined with a Color Doppler System (F31; Hitachi Aloka Medical Ltd.) and 7.5-MHz endorectal probes (Hitachi Aloka Medical Ltd.) in the axial and sagittal sections. Ultrasound equipment was set at the level of background noise. In total, seven sonographers (blinded regarding the mpMRI results; minimum 3 years of experience) of the institute(s) performed endorectal power Doppler with grayscale ultrasound examinations. After the endorectal power Doppler/grayscale ultrasound examinations, a $18 \mathrm{G}$ biopsy needle (BD Surgical, Inc.) was inserted in the location where the most intensive signal had been detected and two cores from the transitional zone, six cores from the peripheral zone, two cores from the hypoechoic region (darker area in the grayscale ultrasound image) and two cores from vascular clustering area (increased number of vessels in power Doppler ultrasound image) (Fig. 1) were collected (5). A total of 15 physicians (blinded regarding the mpMRI results, minimum of three years of experience) of the institute(s) performed the biopsies. Endorectal power Doppler/grayscale ultrasound-guided biopsies adhered to the Standards for Reporting of Diagnostic accuracy studies guidelines (24). To overcome the deformation of ultrasound images obtained by transrectal ultrasound, the ultrasound probe was held against the skin without pressure.

mpMRI/TRUS-guided biopsy. In the same setting as for mpMRI, biopsies were performed using $18 \mathrm{G}$ needle Tru-cut Biopsy Guns (cat. no. 661830, Biocore II BR; Histo, S.A.) under guidance of ultrasound (F31; Hitachi Aloka Medical Ltd.) with a 7.5-MHz endorectal probe (Hitachi Aloka Medical Ltd.). A total of 14 cores were randomly collected from the base, the middle third part, apex (medial and 

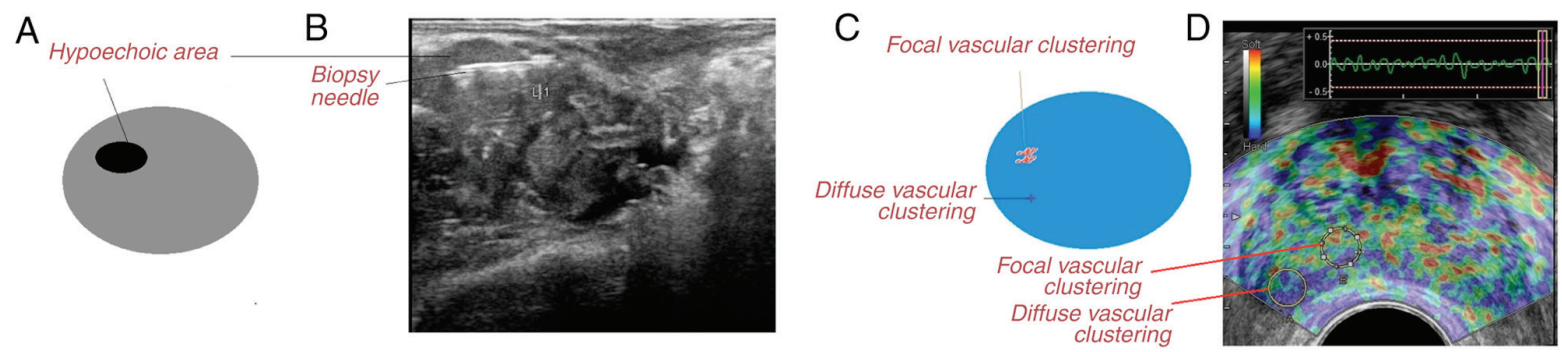

Figure 1. Region of interest for endorectal power Doppler fusion grayscale ultrasound-guided biopsy. (A) Pictorial presentation of the hypoechoic area. (B) Grayscale ultrasound image of the hypoechoic area. (C) Pictorial presentation of vascular clustering. (D) Power Doppler ultrasound image of vascular clustering. A total of 15 physicians (blinded regarding the multiparametric MRI results) had performed the biopsies. All had a minimum of 3 years of experience. The biopsies adhered to the Standards for Reporting of Diagnostic accuracy studies guidelines. L1, left side.

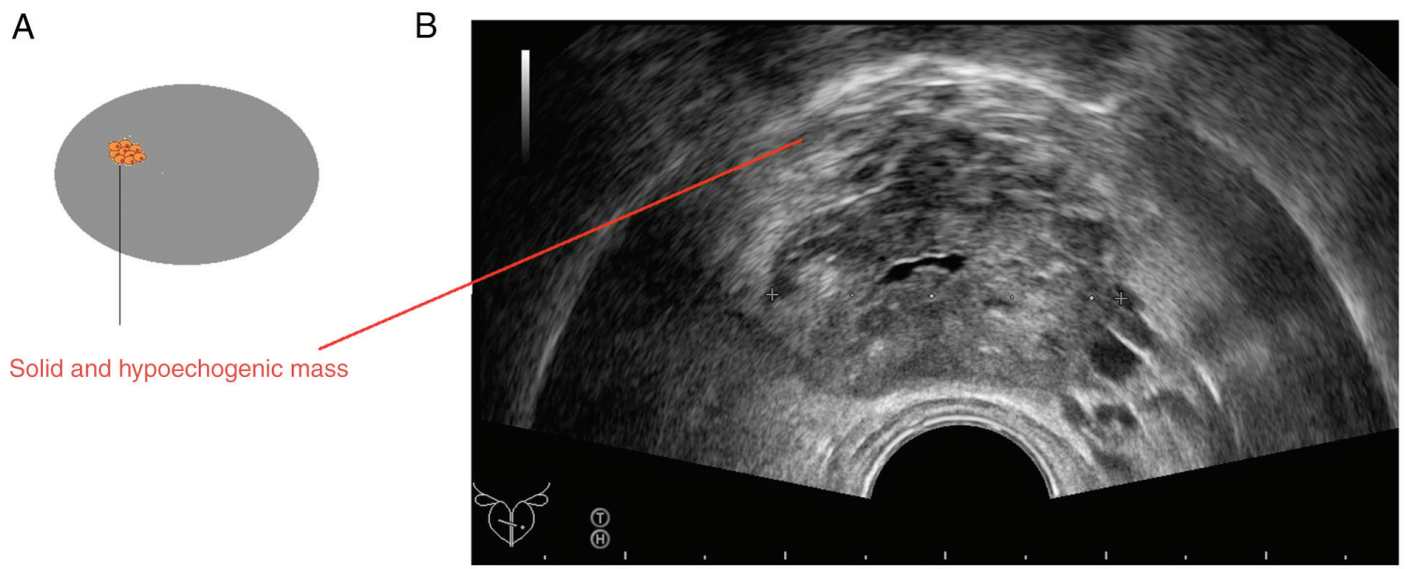

Figure 2. Region of interest for multiparametric MRI fusion with transrectal ultrasound-guided biopsy. (A) Pictorial presentation of the solid and hypoechogenic area. (B) Grayscale ultrasound image of a solid and hypoechogenic area. The biopsies were performed by 15 physicians blinded regarding endorectal power Doppler/grayscale ultrasound-guided biopsies. All had a minimum of 3 years of experience. Biopsies had adhered to the Standards for Reporting of Diagnostic accuracy studies guidelines.

lateral parts) and each side of the transition zone of the prostate. In addition, two cores were collected from solid and hypoechogenic areas (denser mass than usual on the grayscale ultrasound image; Fig. 2) (21). A total of 15 physicians (minimum three years of experience, blinded regarding endorectal power Doppler/grayscale ultrasound-guided biopsies) of the institute(s) performed the biopsies. The mpMRI/TRUS guided biopsies adhered to the guidelines of the Standards of Reporting for MRI-Targeted biopsy studies consortium (25).

For prophylactic purposes, all patients had been prescribed levofloxacin $500 \mathrm{mg}$ twice a day for three days prior to the biopsies and all patients had received a cleansing enema prior to the examinations (26). To control pain, a rectal lidocaine jelly was applied $30 \mathrm{~min}$ prior to the biopsies and afterwards, oral $50 \mathrm{mg}$ diclofenac with $500 \mathrm{mg}$ paracetamol was prescribed twice a day for two days (5). Anticholinergic drugs (oxybutynin, $5 \mathrm{mg}$ orally 2-3 times a day) were given to the patients prior to the biopsies to overcome difficulties in diagnosis due to reactions including pelvic muscle pressure and pelvic muscle contraction.

Pathological analysis. The biopsy lesions were preserved in $10 \%$ formalin and sent to a laboratory for histopathology purposes. All samples were paraffin-embedded and slides were prepared. The slides were stained with hematoxylin and eosin. Each slide was examined under a light microscope (Olympus) by urologic pathologists. A total of 13 pathologists (minimum three years of experience) of the institute(s) examined the histopathological results. Each histopathological specimen was assigned primary and secondary Gleason scores (Fig. 3), numbers of cores positive for cancer and the percentage of cores with cancer $(21,27)$. Samples with Gleason scores $\geq 3+4$ (defined as most of the tumor being of grade 3 and the next-largest section of the tumor being grade 4) were considered as prostate cancer $(21,28)$.

Robotic-assisted laparoscopic radical prostatectomy. After obtaining histopathological results of the two types of biopsies, the urologists performed robotic-assisted laparoscopic radical prostatectomy in males aged $<70$ years with Gleason scores of $\geq 3+4$ in any one of the biopsy reports (29). During the surgical procedure, the prostate and surrounding tissues were removed (9). A total of 17 urologists (minimum three years of experience) of the institute(s) performed the prostatectomy. A single-surgeon approach was used. Details on prostatectomy were reported in line with the Strengthening the Reporting of Cohort Studies in Surgery criteria (30). 


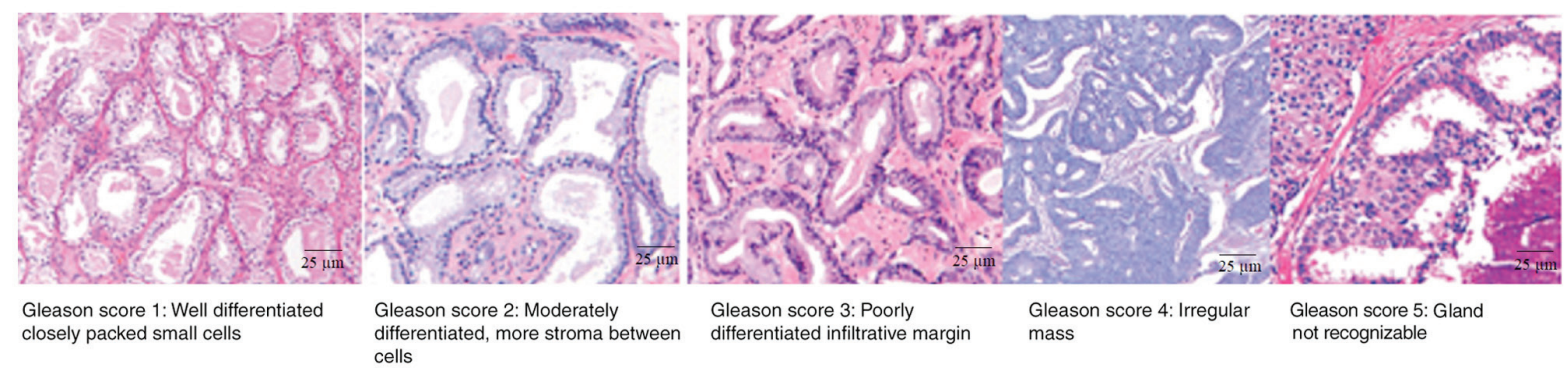

Figure 3. Representative histopathological images of biopsy specimens with different Gleason scores. The histopathological results were examined by 13 pathologists with a minimum of 3 years of experience. The pathological analysis was performed according to the Stanford technique. The original length of all histopathological images was $5 \mathrm{~mm}$ (scale bar, $25 \mu \mathrm{m}$ ).

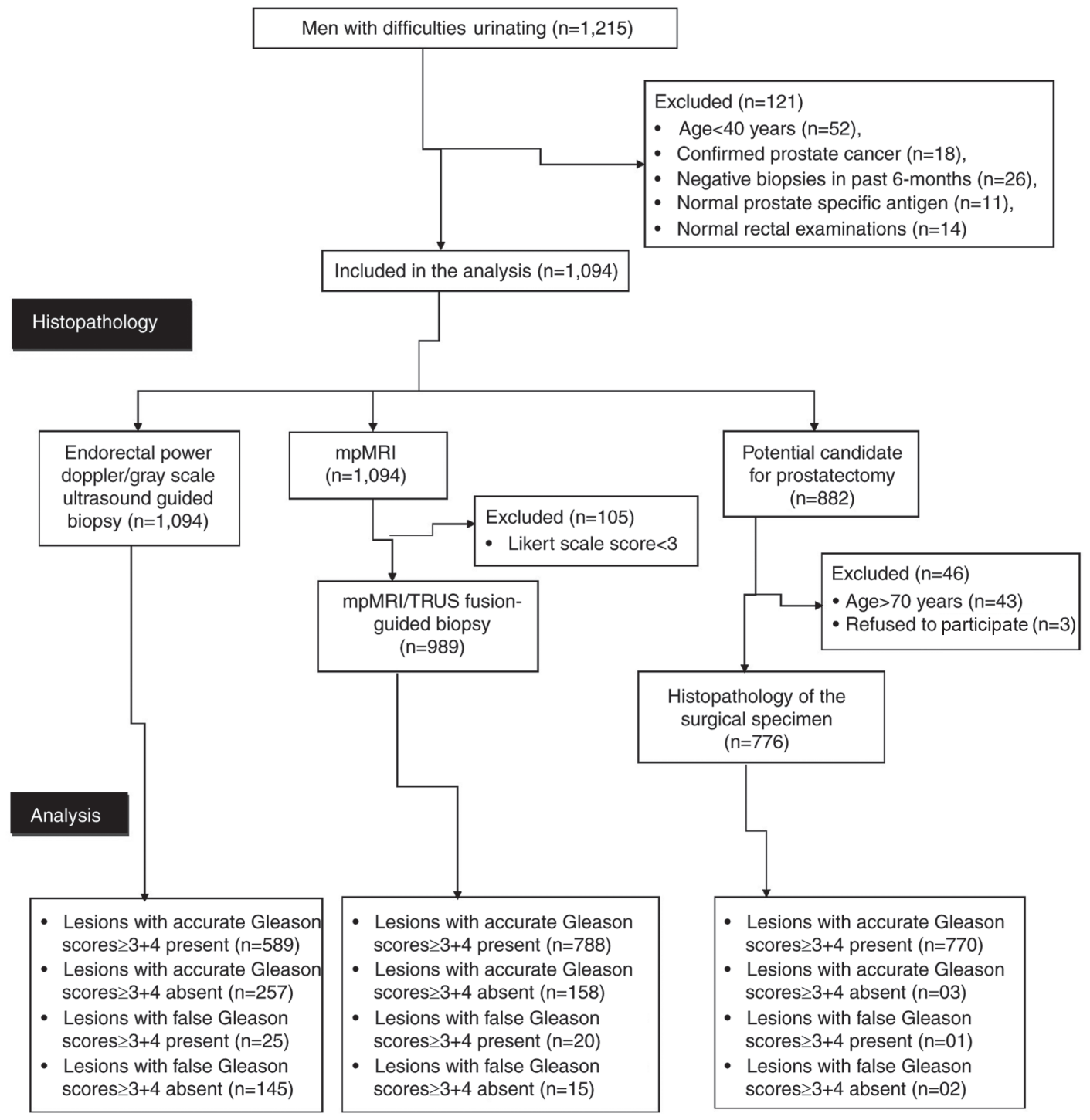

Figure 4. Flow diagram of the study. mpMRI was performed by 5 radiologists who also assigned Likert scale score to each lesion. Endorectal power doppler/grayscale ultrasound-guided biopsies were performed by 15 physicians. mpMRI/TRUS-guided biopsies were also performed by physicians. The histopathological results were examined by 13 pathologists. All had a minimum of 3 years of experience and were blinded regarding the results obtained with the other methods. A Gleason score of $\geq 3+4$ was defined as most of the tumor being grade 3 and the next-largest section of the tumor being grade 4 . mpMRI/TRUS, multiparametric MRI fusion with transrectal ultrasound.

Histopathology of the surgical specimen. Sampling of the resected prostate was performed using the Stanford technique. Transverse (4-5 $\mathrm{mm})$ and sagittal $(6-7 \mathrm{~mm})$ sections from apex to base were taken and subjected to pathological examination (9). Gleason scores were recorded $(21,27)$. Specimens with Gleason scores of $\geq 3+4$ were considered as prostate 
cancer $(21,28)$. A total of 10 pathologists (minimum three years of experience; blinded regarding radiological examinations) of the institute(s) examined the histopathological results.

Prostatectomy decision analysis. Decision curve analysis was performed to evaluate the prostatectomy decision in the subjects enrolled as per Eq. i (31):

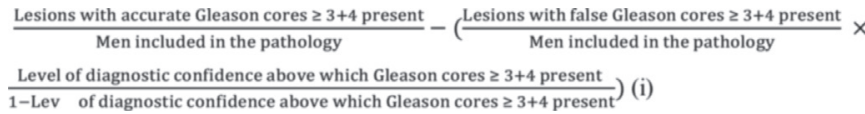

Statistical analysis. InStat Software for Windows (version 3.0; GraphPad Inc.) was used for statistical analysis. Differences between groups in discrete variables were analyzed using Fisher's exact test (21). Continuous data are presented as the mean \pm standard deviation. Linear weighted $\mathrm{k}$ coefficients were determined to evaluate interobserver agreement (poor agreement, $\mathrm{k} \leq 0.40$; moderate agreement, $0.4>\mathrm{k} \leq 0.60$; and substantial agreement, $\mathrm{k}>0.60)$ (22). All results were considered significant at a confidence level of $99 \%$.

\section{Results}

Patients. Among included patients, 52 patients had an age of $<40$ years, 18 had confirmed prostate cancer, 26 had negative biopsy reports in the past 6 months, 11 had normal prostate-specific antigen values and 14 had normal rectal examinations, and were therefore excluded from the analysis. Finally, the data of 1,094 subjects were included in the study. The flow diagram of the study is presented in Fig. 4.

Medical history and demographics. The demographic parameters of the cohort are provided in Table I. Among the patients (age range, 41-91 years; mean age: 69.45 \pm 8.47 years) enrolled, 90\% were Han Chinese, $9 \%$ were Mongolians and $1 \%$ were Tibetans. Furthermore, $48 \%$ of the subjects had a body mass index of $<25 \mathrm{~kg} / \mathrm{m}^{2}, 33 \%$ of $25-30 \mathrm{~kg} / \mathrm{m}^{2}$ and $19 \%$ of $>30 \mathrm{~kg} / \mathrm{m}^{2}$. A total of 293 patients were obese (definition, waist circumference $>490 \mathrm{~cm}$ ). In addition, $32 \%$ of the subjects had diabetes and $18 \%$ had hypertension.

Pathological analysis. By using endorectal power Doppler/grayscale ultrasound-guided biopsies following pathological analysis, Gleason scores of $\geq 3+4$ were determined in 589 patients. On mpMRI, lesions were identified in 105 subjects with a Likert scale score of $<3$. Therefore, the other 989 patients were subjected to mpMRI/TRUS-guided biopsies. mpMRI/TRUS-guided biopsies followed by pathological analysis revealed Gleason scores of $\geq 3+4$ in 808 patients. Among those subjects with a Likert scale score of $<3$ ( $n=105)$ who were not subjected to mpMRI/TRUS-guided biopsy, 14 had reported Gleason scores of $\geq 3+4$ determined from endorectal power Doppler/grayscale ultrasound-guided biopsies. Therefore, a total of 822 patients had Gleason scores of $\geq 3+4$ in either of the biopsy reports. Among them, 43 patients had an age of $\geq 70$ years (as per institutional guidelines for surgery). Therefore, the urologists did not
Table I. Demographical characteristics of the male patients enrolled $(n=1,094)$.

\begin{tabular}{|c|c|}
\hline Characteristic & Value \\
\hline \multicolumn{2}{|l|}{ Age (years) } \\
\hline Range & $41-91$ \\
\hline Mean $\pm \mathrm{SD}$ & $69.45 \pm 8.47$ \\
\hline \multicolumn{2}{|l|}{ Ethnicity } \\
\hline Han Chinese & $983(90)$ \\
\hline Mongolian & $102(9)$ \\
\hline Tibetan & $9(1)$ \\
\hline \multicolumn{2}{|c|}{ Serum prostate-specific antigen $(\mathrm{ng} / \mathrm{ml})$} \\
\hline 40-49 years & $7.12 \pm 1.01$ \\
\hline $50-59$ years & $9.01 \pm 1.22$ \\
\hline $60-69$ years & $10.22 \pm 1.55$ \\
\hline$\geq 70$ years & $11.19 \pm 1.89$ \\
\hline \multicolumn{2}{|l|}{ Body mass index $\left(\mathrm{kg} / \mathrm{m}^{2}\right)$} \\
\hline$<25$ & $523(48)$ \\
\hline $25-30$ & $359(33)$ \\
\hline$>30$ & $212(19)$ \\
\hline Diabetes $^{\mathrm{a}}$ & $347(32)$ \\
\hline Hypertension $^{\mathrm{b}}$ & $201(18)$ \\
\hline \multicolumn{2}{|l|}{ Manual rectal examination } \\
\hline Hardness & $414(38)$ \\
\hline Lumps & $343(31)$ \\
\hline Enlarged prostate & $337(31)$ \\
\hline \multicolumn{2}{|l|}{ Central obesity } \\
\hline No & $801(73)$ \\
\hline Yes & $293(27)$ \\
\hline Alcohol abuse & $45(4)$ \\
\hline Chronic urinary tract infection & $12(1)$ \\
\hline
\end{tabular}

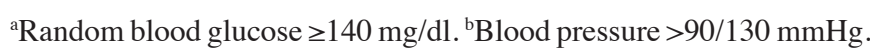
${ }^{\mathrm{c}}$ Central obesity was defined as a waist circumference of $>490 \mathrm{~cm}$, while a waist circumference of $\leq 90 \mathrm{~cm}$ was defined as no central obesity. Constant variables are presented as n (\%) and continuous data are presented as the mean \pm SD unless otherwise specified. SD, standard deviation. Normal value of body mass index for Chinese men is $25 \pm 2 \mathrm{~kg} / \mathrm{m}^{2}$.

offer them any prostatectomy and 3 further patients refused to undergo surgery. Finally, a total of 776 subjects underwent prostatectomy and all resected prostates were subjected to pathological analysis (Fig. 5).

Diagnostic parameters. Endorectal power Doppler/grayscale ultrasound-guided biopsy had a lower sensitivity than histopathology of the surgical specimen (0.93 vs. $1.00, \mathrm{P}<0.0001)$ but mpMRI/TRUS-guided biopsy had the same sensitivity as that of the histopathology of the surgical specimen $(0.99$ vs. $1.00, \mathrm{P}=0.02$ ) and negligible inconclusive results (8 vs. $0, \mathrm{P}=0.024)$. Accuracy was in the order of histopathology of the surgical specimen (1.00)>mpMRI/TRUS-guided biopsy $(0.944)>$ endorectal power Doppler/grayscale ultrasound-guided biopsy (0.783). However, compared with 
Table II. Diagnostic parameters determined with various pathological methods.

Parameters

Either biopsy results

Histopathology of the

( $n=1,094$ subjects)

surgical specimen $(n=776)$

P-value

Lesions with accurate Gleason scores $\geq 3+4$ present

808 (74)

249 (22)

14 (1)

Lesions with false Gleason scores $\geq 3+4$ present

Lesions with false Gleason scores $\geq 3+4$ absent

$15(2)^{\mathrm{a}}$

$8(1)^{\mathrm{a}}$

0.964

Accuracy

$0.99^{\mathrm{a}}$

$770(99.2)$
$03(0.4)$
$01(0.1)$
$02(0.3)$
$00(0)$
1
1

$<0.0001$

$<0.0001$

0.006

0.012

0.024

$<0.0001$

0.024

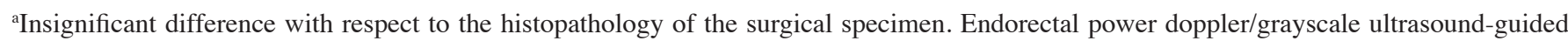
biopsies (Standards for Reporting of Diagnostic accuracy studies guidelines) were performed by 15 physicians. Five radiologists performed mpMRI and assigned a Likert scale score to each lesion. mpMRI/transrectal ultrasound fusion-guided biopsies (in line with the guidelines of the Standards of Reporting for MRI-Targeted biopsy studies consortium) were performed by 15 physicians and 13 pathologists examined the histopathological results (Stanford technique). All had a minimum of 3 years of experience and were blinded regarding the results obtained with the other methods. A Gleason score of $\geq 3+4$ was defined as most of the tumor being grade 3 and the next-largest section of the tumor being grade 4 . Constant variables are presented as $\mathrm{n}(\%)$. Fisher's exact test was used for statistical analysis. $\mathrm{P}<0.01$ was considered to indicate statistical significance. mpMRI, multiparametric MRI.

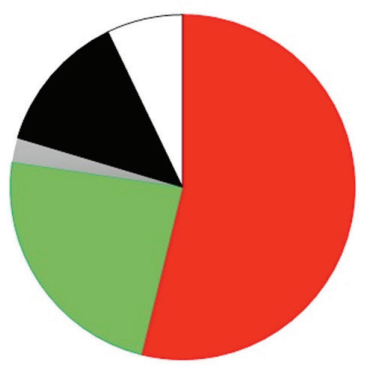

Endorectal power doppler/gray scale ultrasound guided biopsy

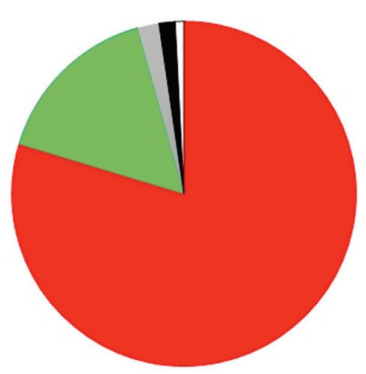

mpMRI/TRUS fusion-guided biopsies

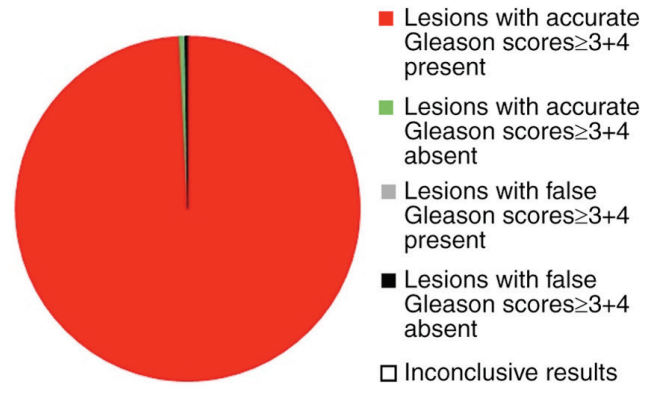

Histopathology of the surgical specimen

Figure 5. Results of the pathological analysis. Endorectal power doppler/grayscale ultrasound-guided biopsies (Standards for Reporting of Diagnostic accuracy studies guidelines) were performed by 15 physicians, mpMRI/TRUS-guided biopsies (in line with the guidelines of the Standards of Reporting for MRI-targeted biopsy studies consortium) were performed by 15 physicians and 13 pathologists examined the histopathological results (Stanford technique). All had a minimum of 3 years of experience and were blinded regarding the results obtained with the other methods. A Gleason score of $\geq 3+4$ was defined as most of the tumor being grade 3 and the next-largest section of the tumor being grade 4. mpMRI/TRUS, multiparametric MRI fusion with transrectal ultrasound.

the histopathological examination of the surgical specimen, the combined results of the two biopsies exhibited insignificant false-negative results $(\mathrm{P}=0.012)$, inconclusive results $(\mathrm{P}=0.024)$ and sensitivity $(\mathrm{P}=0.024)$ but higher accuracy (Table II).

Interobserver agreement. Compared with final results adopted, all evaluations had a moderate linear-weighted agreement (0.4> k $\leq 0.60$; Table III).

Prostatectomy decision analysis. The working area that detects at least one lesion with Gleason scores $\geq 3+4$ for endorectal power Doppler/grayscale ultrasound-guided biopsies, mpMRI/TRUS-guided biopsies, and combinations of endorectal power Doppler/grayscale ultrasound-guided biopsies and mpMRI/TRUS-guided biopsies was 0.330-0.920, 0.180-0.935 and 0.170-0.965, respectively. Above 0.920,
0.935 and 0.965 , endorectal power Doppler/grayscale ultrasound-guided biopsies, mpMRI/TRUS-guided biopsies and combinations of endorectal power Doppler/grayscale ultrasound-guided biopsies and mpMRI/TRUS-guided biopsies had the risk of overdiagnosis (Fig. 6).

\section{Discussion}

The present study indicated that mpMRI/TRUS-guided biopsies had a higher working area that detects Gleason scores $\geq 3+4$ at least one time in the collected lesion. In addition, the same sensitivity, negligible inconclusive results and high accuracy to those of the histopathology analysis of the surgical specimen were obtained. The results of the study were in line with those of previous studies $(8,9,11-13,15,19,21,23)$. mpMRI/TRUS-guided biopsy promises the detection of clinically significant prostate cancer(s). 
Table III. Interobserver agreement for the different evaluation methods.

\begin{tabular}{lcccc}
\hline & $\begin{array}{c}\text { Endorectal power } \\
\text { doppler/grayscale } \\
\text { Parameter }\end{array}$ & mpMRI & $\begin{array}{c}\text { mpMI/TRUS-guided } \\
\text { biopsies }\end{array}$ & Pathological analysis \\
\hline Adhered guideline & STARD & Likert scale score & START & Stanford technique \\
Evaluators (n) & 15 & 5 & 15 & 13 \\
k value & 0.51 & 0.53 & 0.56 & 0.49 \\
\hline
\end{tabular}

Poor agreement, $\mathrm{k} \leq 0.40$; moderate agreement, $0.4>\mathrm{k} \leq 0.60$; substantial agreement, $\mathrm{k}>0.60$. STARD, Standards for Reporting of Diagnostic accuracy studies; START, Standards of Reporting for MRI-Targeted biopsy studies; mpMRI/TRUS, multiparametric MRI fusion with transrectal ultrasound.

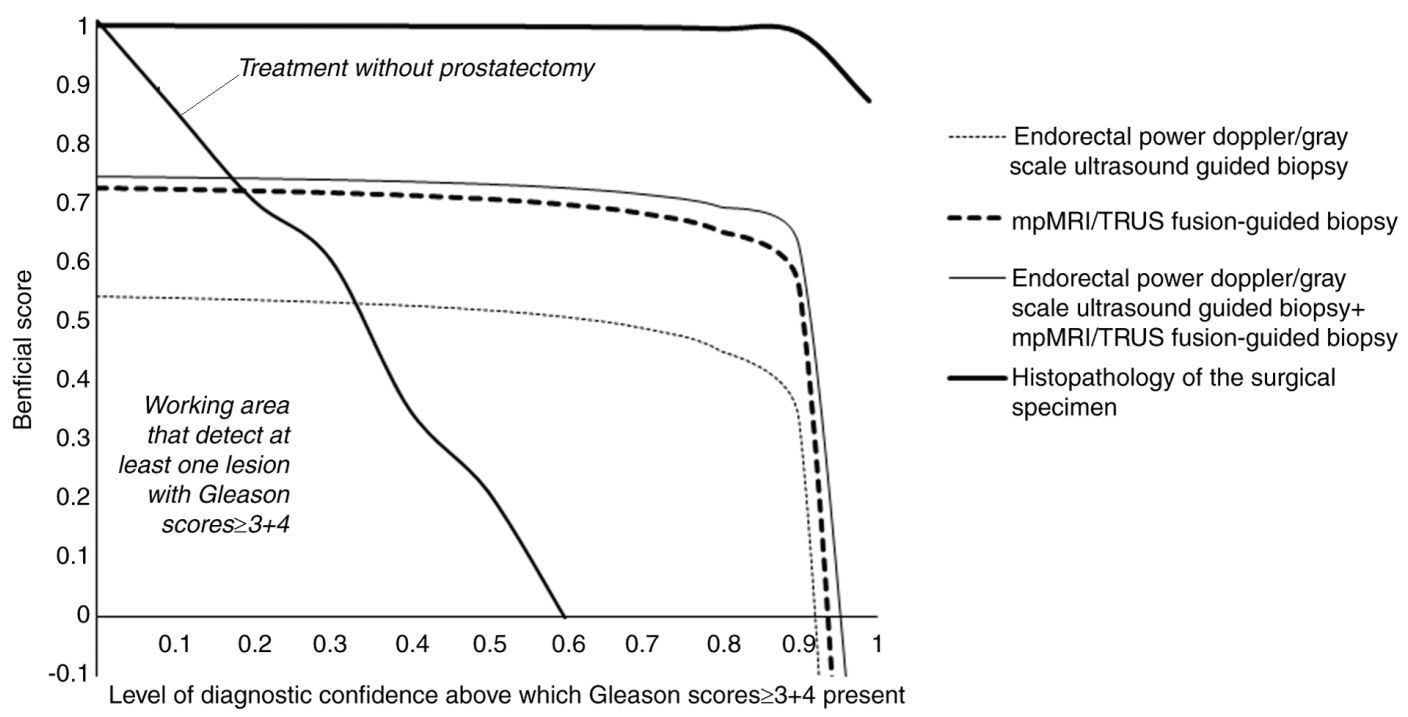

Figure 6. Prostatectomy decision analysis. Treatment without proctectomy was an imaginary option, including radiation therapy and/or medication. Endorectal power doppler/grayscale ultrasound-guided biopsies (Standards for Reporting of Diagnostic accuracy studies guidelines) were performed by 15 physicians. Five radiologists performed mpMRI and assigned a Likert scale score to each lesion. mpMRI/TRUS-guided biopsies (in line with the guidelines of the Standards of Reporting for MRI-Targeted biopsy studies consortium) were performed by 15 physicians and 13 pathologists examined the histopathological results (Stanford technique). All had a minimum of 3 years of experience and were blinded regarding the results obtained with the other methods. Prostatectomy had been reported in line with the Strengthening the Reporting of Cohort Studies in Surgery criteria. A Gleason score of $\geq 3+4$ was defined as most of the tumor being grade 3 and the next-largest section of the tumor being grade 4. mpMRI/TRUS, multiparametric MRI fusion with transrectal ultrasound. Working area of diagnosis: Space between two cut-off limits to detect prostate cancer at least one time.

The negative predictive value of mpMRI/TRUS-guided biopsy was then assessed. During the study, $105(10 \%)$ of the patients had a Likert scale score of $<3$ determined by mpMRI and mpMRI/TRUS-guided biopsies identified lesions with Gleason scores $<3+4$ in 173 (16\%) of patients but they had abnormal rectal examinations and elevated prostate-specific antigen. In addition, among the patients with a Likert scale score of $<3,14$ had Gleason scores of $\geq 3+4$ determined by endorectal power Doppler/grayscale ultrasound-guided biopsies. Furthermore, analysis of the mpMRI/TRUS-guided biopsies of the lesions of 20 patients gave a false-positive result, as no prostate cancer was detected by histopathological analysis of the surgical specimen. Seminal vesicle invasion may be more precisely detected by histopathology compared with mpMRI (32). Low-grade tumors are isointense and not detected on T2WI. In addition, tumors in the transition zone are more difficult to detect than those in the peripheral zone (33).
Post-irradiation tissue damage, scars, atrophy and prostatitis may also be assumed to be prostate cancer on mpMRI (32). The results of the present study indicate a moderate performance of mpMRI/TRUS-guided biopsy in the detection of prostate cancer.

Compared to histopathology of the surgical specimen, endorectal power Doppler/grayscale ultrasound-guided biopsies had a moderate working area that detects Gleason scores $\geq 3+4$ at least one time in the collected lesions, lower sensitivity and accuracy, and a higher number of inconclusive results $(78 ; 7 \%)$ and false-negative results $(145 ; 13 \%)$. The results of the present study were in line with those of previous studies $(5,16,34,35)$. Power Doppler US is not able to differentiate cancerous vascular clustering lesions from hypervascular inflammation (5). Endorectal power Doppler/grayscale ultrasound-guided biopsy appears to have a barely sufficient diagnostic performance in the detection of prostate cancer. 
According to the present results, combination of the two biopsies provided a sensitivity of 0.99 , accuracy of 0.964 , the highest working area that detects at least one lesion with Gleason scores $\geq 3+4$, as well as insignificant false-negative lesions and inconclusive results compared with the results of the histopathology of the surgical specimen. However, the Chinese Guidelines on Urologic Diseases in the newest 2014 version do not recommend this combination of these two biopsies for detection of prostate cancer (36). The next version of the Chinese Guidelines on Urologic Diseases requires to be updated.

Of note, the present study has several limitations. For instance, survival data during the follow-up were not reported. Furthermore, there was a lack of randomization. In addition, the treatment strategies for 46 patients, to whom the urologists had not offered any prostatectomy $(n=43)$, those who refused to undergo surgery $(n=3)$, and those with a low and intermediate risk, were not included. Two types of biopsy were performed, which was not recommended in a clinical setting. In addition, the results of Chinese populations may not be comparable to those from other regions of the world.

In conclusion, mpMRI/TRUS-guided biopsy was indicated to have a moderate performance and endorectal power Doppler/grayscale ultrasound-guided biopsy had a scant performance for decision-making regarding prostatectomy. It is recommended that the health department of the P.R. China releases a new algorithm for the detection of prostate cancer in Chinese males in its new Guidelines on Urologic Diseases.

\section{Acknowledgements}

Not applicable.

\section{Funding}

No funding received.

\section{Availability of data and materials}

The datasets used and/or analyzed during the current study are available from the corresponding author on reasonable request.

\section{Authors' contributions}

All authors have reviewed and approved the manuscript submitted for publication. $\mathrm{ZH}$ was project administrator and contributed to the data curation, literature review and supervision of the study. ZY contributed to the conceptualization, literature review and resources of the study. LL contributed to the conceptualization, validation and literature review included in the study. XX contributed to the resources, data curation, formal analysis, literature review, and editing of the manuscript for intellectual content. JY contributed to the formal analysis, software processing and literature review of the study. WH contributed to the investigation, literature review, software and supervision of the study. JC contributed to the supervision, validation, formal analysis and literature review included in the study. YK contributed to the formal analysis and literature review, as well as drafting reviewing and editing of the manuscript for intellectual content. The authors agreed to be accountable for all aspects of work ensuring integrity and accuracy.

\section{Ethical approval and consent to participate}

The protocol of the study (DPH/CL/04/15 dated 12 January 2013) was approved by the review board of Southern Medical University. The study adhered to China, Strengthening the Reporting of Observational studies in Epidemiology statement and the 2008 Helsinki Declaration. An informed consent form had been signed by each of the patients enrolled prior to participation regarding pathology, radiology, biopsies, surgeries (if required), and to undergo an additional procedure for research purposes only.

\section{Patient consent for publication}

Not applicable.

\section{Competing interests}

The authors declare that they have no competing interests.

\section{References}

1. Egidi MG, Cochetti G, Serva MR, Guelfi G, Zampini D, Mechelli L and Mearini E: Circulating microRNAs and kallikreins before and after radical prostatectomy: Are they really prostate cancer markers? Biomed Res Int 2013: 241780, 2013.

2. Pan J, Xue W, Sha J, Yang H, Xu F, Xuan H, Li D and Huang Y: Incidental prostate cancer at the time of cystectomy: The incidence and clinicopathological features in Chinese patients. PLoS One 9: e94490, 2014.

3. Ferlay J, Soerjomataram I, Dikshit R, Eser S, Mathers C, Rebelo M, Parkin DM, Forman D and Bray F: Cancer incidence and mortality worldwide: Sources, methods and major patterns in GLOBOCAN 2012. Int J Cancer 136: E359-E386, 2015.

4. Wong MC, Goggins WB, Wang HH, Fung FD, Leung C, Wong SY, Ng CF and Sung JJ: Global incidence and mortality for prostate cancer: Analysis of temporal patterns and trends in 36 countries. Eur Urol 70: 862-874, 2016.

5. Kahraman T, Cubuk R, Sinanoglu O, Tasalı N, Ozarar M and Saydam B: Comparison of power Doppler ultrasound with gray scale transrectal ultrasound in predicting cancer positive prostate biopsy cores. Eurasian J Med 42: 81-85, 2010.

6. Guelfi G, Cochetti G, Stefanetti V, Zampini D, Diverio S, Boni A and Mearini E: Next generation sequencing of urine exfoliated cells: An approach of prostate cancer microRNAs research. Sci Rep 8: 7111, 2018.

7. Zhang J, Ma M, Nan X and Sheng B: Obesity inversely correlates with prostate-specific antigen levels in a population with normal screening results of prostate cancer in northwestern China. Braz J Med Biol Res 49: pii: S0100-879X2016000800704, 2016.

8. Kaufmann S, Kruck S, Kramer U, Gatidis S, Stenzl A, Roethke M, Scharpf M and Schilling D: Direct comparison of targeted MRI-guided biopsy with systematic transrectal ultrasound-guided biopsy in patients with previous negative prostate biopsies. Urol Int 94: 319-325, 2015.

9. Baco E, Ukimura O, Rud E, Vlatkovic L, Svindland A, Aron M, Palmer S, Matsugasumi T, Marien A, Bernhard JC, et al: Magnetic resonance imaging-transectal ultrasound image-fusion biopsies accurately characterize the index tumor: Correlation with step-sectioned radical prostatectomy specimens in 135 patients. Eur Urol 67: 787-794, 2015.

10. Cochetti G, Poli G, Guelfi G, Boni A, Egidi MG and Mearini E: Different levels of serum microRNAs in prostate cancer and benign prostatic hyperplasia: Evaluation of potential diagnostic and prognostic role. Onco Targets Ther 9: 7545-7553, 2016.

11. Zhang Q, Wang W, Zhang B, Shi J, Fu Y, Li D, Guo S, Zhang S, Huang $\mathrm{H}$, Jiang $\mathrm{X}$, et al: Comparison of free-hand transperineal mpMRI/TRUS fusion-guided biopsy with transperineal 12-core systematic biopsy for the diagnosis of prostate cancer: A single-center prospective study in China. Int Urol Nephrol 49: 439-448, 2017. 
12. Siddiqui MM, Rais-Bahrami S, Turkbey B, George AK, Rothwax J, Shakir N, Okoro C, Raskolnikov D, Parnes HL, Linehan WM, et al: Comparison of MR/ultrasound fusion-guided biopsy with ultrasound-guided biopsy for the diagnosis of prostate cancer. JAMA 313: 390-397, 2015.

13. Borkowetz A, Platzek I, Toma M, Laniado M, Baretton G, Froehner M, Koch R, Wirth M and Zastrow S: Comparison of systematic transrectal biopsy to transperineal magnetic resonance imaging/ultrasound-fusion biopsy for the diagnosis of prostate cance. BJU Int 116: 873-879, 2015.

14. Pepe P, Garufi A, Priolo G and Pennisi M: Can MRI/TRUS fusion targeted biopsy replace saturation prostate biopsy in the re-evaluation of men in active surveillance? World J Urol 34 1249-1253, 2016

15. Lian H, Zhuang J, Wang W, Zhang B, Shi J, Li D, Fu Y, Jiang X, Zhou W and Guo H: Assessment of free-hand transperineal targeted prostate biopsy using multiparametric magnetic resonance imaging-transrectal ultrasound fusion in Chinese men with prior negative biopsy and elevated prostate-specific antigen. BMC Urol 17: 52, 2017

16. Ezquer A, Ortega Hrescak MC, Sanagua C, Roggia-Rebullida P, López R, Cenice F, Veglia FH, Veglia F and Fernández A: Transrectal doppler ultrasound during prostate biopsy: Clinical utility and limitations. Actas Urol Esp 39: 13-19, 2015 (In English, Spanish).

17. Cochetti G, Boni A, Barillaro F, Pohja S, Cirocchi R and Mearini E: Full neurovascular sparing extraperitoneal robotic radical prostatectomy: Our experience with PERUSIA technique. J Endourol 31: 32-37, 2017

18. Boni A, Cochetti G, Del Zingaro M, Paladini A, Turco M, Rossi de Vermandois JA and Mearini E: Uroflow stop test with electromyography: A novel index of urinary continence recovery after RARP. Int Urol Nephrol 51: 609-615, 2019.

19. Zhu G and Wang Q: Comparisons between magnetic resonance/ultrasound fusion-guided biopsy and standard biopsy in the diagnosis of prostate cancer: A prospective cohort study. Medicine (Baltimore) 97: e11962, 2018

20. Huang M, Lin Y, Xu A, Uhlman M, Deng X, Lin X, Wu S, Diao P, Xie K and Tang P: Percent free prostate-specific antigen does not improve the effectiveness of prostate cancer detection in Chinese men with a prostate-specific antigen of 2.5-20.0 ng/ml: A multicenter study. Med Oncol 31: 925, 2014.

21. Mariotti GC, Falsarella PM, Garcia RG, Queiroz MRG, Lemos GC and Baroni RH: Incremental diagnostic value of targeted biopsy using mpMRI-TRUS fusion versus 14-fragments prostatic biopsy: A prospective controlled study. Eur Radiol 28: 11-16, 2018.

22. Equator Network: The Standards for Reporting of Diagnostic accuracy studies guidelines. Available from: http://www.equator-network.org/reporting-guidelines/stard/. Accessed September 6, 2019.

23. Barentsz JO, Richenberg J, Clements R, Choyke P, Verma S, Villeirs G, Rouviere O, Logager V and Fütterer JJ; European Society of Urogenital Radiology: ESUR prostate MR guidelines 2012. Eur Radiol 22: 746-757, 2012
24. Rosenkrantz AB, Kim S, Lim RP, Hindman N, Deng FM, Babb JS and Taneja SS: Prostate cancer localization using multiparametric MR imaging: Comparison of prostate imaging reporting and data system (PI-RADS) and Likert scales. Radiology 269: 482-492, 2013.

25. Costa DN, Lotan Y, Rofsky NM, Roehrborn C, Liu A, Hornberger B, Xi Y, Francis F and Pedrosa I: Assessment of prospectively assigned Likert scores for targeted magnetic resonance imaging-transrectal ultrasound fusion biopsies in patients with suspected prostate cancer. J Urol 195: 80-87, 2016.

26. Moore CM, Kasivisvanathan V, Eggener S, Emberton M, Fütterer JJ, Gill IS, Grubb Iii RL, Hadaschik B, Klotz L, Margolis DJ, et al: Standards of reporting for MRI-targeted biopsy studies (START) of the prostate: Recommendations from an International working group. Eur Urol 64: 544-552, 2013.

27. Dell'atti L: Prostatic abscess after transrectal ultrasound-guided prostate biopsy. Case report. G Chir 39: 260-262, 2013.

28. Ozok HU, Sagnak L, Tuygun C, Oktay M, Karakoyunlu N, Ersoy H and Alper M: Will the modification of the Gleason grading system affect the urology practice? Int J Surg Pathol 18: 248-254, 2010.

29. Labanaris AP, Witt JH and Zugor V: Robotic-assisted radical prostatectomy in men $\geq 75$ years of age. Surgical, oncological and functional outcomes. Anticancer Res 32: 2085-2089, 2012.

30. Agha RA, Borrelli MR, Vella-Baldacchino M, Thavayogan R and Orgill DP; STROCSS Group: The STROCSS statement: Strengthening the reporting of Cohort studies in surgery. Int $\mathrm{J}$ Surg 46: 198-202, 2017.

31. Fitzgerald M, Saville BR and Lewis RJ: Decision curve analysis. JAMA 13: 409-410, 2015

32. Bjurlin MA, Mendhiratta N, Wysock JS and Taneja SS: Multiparametric MRI and targeted prostate biopsy: Improvements in cancer detection, localization, and risk assessment. Cent European J Urol 69: 9-18, 2016.

33. Tan CH, Hobbs BP, Wei W and Kundra V: Dynamic contrast-enhanced MRI for the detection of prostate cancer: Meta-analysis. Am J Roentgenol 204: W439-W448, 2015.

34. Sohail SK, Sarfraz R, Imran M, Khan NA and Yusuf NW: Power doppler ultrasonography guided and random prostate biopsy in prostate cancer diagnosis-a comparative study. J Pak Med Assoc 65: 65-68, 2015

35. Delgado Oliva F, Arlandis Guzman S, Bonillo García M, Broseta Rico E and Boronat Tormo F: Diagnostic performance of power doppler and ultrasound contrast agents in early imaging-based diagnosis of organ-confined prostate cancer: Is it possible to spare cores with contrast-guided biopsy? Eur J Radiol 85: 1778-1785, 2016

36. Na YQ, Ye ZQ, Sun YH and Sun G: Chinese Guidelines on Urologic Diseases. PR China Helath Department. People's Medical Publishing House, Beijing, 2015 (In Chinese).

This work is licensed under a Creative Commons Attribution-NonCommercial-NoDerivatives 4.0 International (CC BY-NC-ND 4.0) License. 